\title{
UK Primary Hyperparathyroidism Clinical Practice Audit
}

\section{A Young Diabetologist's \& Endocrinologist's Forum (YDEF) Project}

\section{Background:}

Primary hyperparathyroidism (PHPT) is a common endocrine disorder associated with excess morbidity and mortality, but there exists marked variation in it's investigation, work up and management in clinical practice.

- The $3^{\text {rd }}$ International Workshop on the management of asymptomatic PHPT (2009) has developed clear evidence based guidance, however several studies have shown that in both the US and Europe there are significant differences in the way that individuals are assessed and treated when compared to these standards of care.

- A national study was set up via the YDEF to explore how UK practice fared in comparison to the workshop guidance, as well as to constructively critique the guidance.

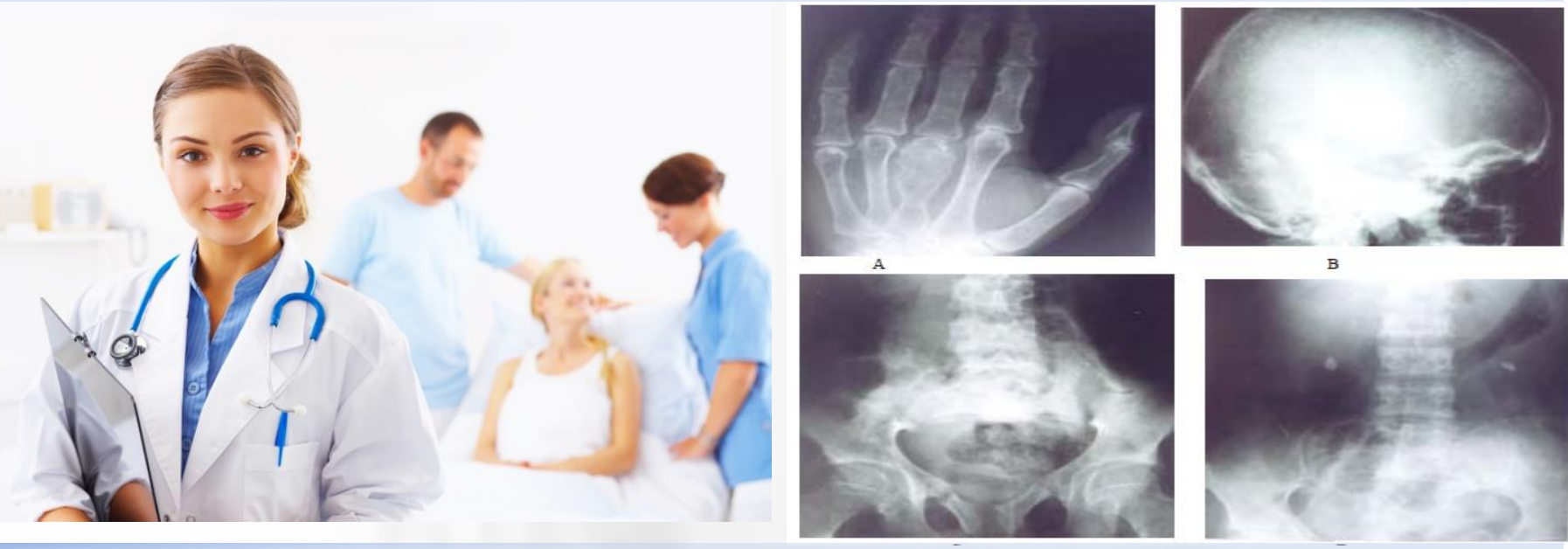

eg. National Survey on the management of PHPT by Swiss Endocrinologists

\section{Guideline recommendations:}

Indications for surgery; Age less than $50 \mathrm{yrs}$; Calcium $>1.0 \mathrm{mg} / \mathrm{dl}$ ( 0 . $25 \mathrm{mmol} / \mathrm{I})>$ than ULN; eGFR $<60 \mathrm{mls} / \mathrm{min}$; BMD:T-score $<-2.5$ at any site (or previous fragility \#); logistical reasons

Localisation of abnormal parathyroid glands - "this should only form part of a pre-operative assessment and is not indicated in the initial diagnosis of hyperparathyroidism"

Criticisms of the Guidelines; 2009 guidance is good, but;

- Pro-surgery

- Don't include useful advice on medical therapies

- Doesn't include information on bone turnover markers

- Forearm DEXA isn't done by many centres - does it add much?

- New data on cardiovascular manifestations and neuropsychological problems have emerged since 2009.

- No discussion of normocalcaemic hyperparathyroidism

- No inclusion of relevance of PTH level itself

Since completion of this audit, the $4^{\text {th }}$ international guidelines have been published which take on board some of the issues that we have identified.

\section{Methods:}

This study was a multi-centre, retrospective, observational audit of cases with confirmed PHPT (normal Vit D levels and renal function).

- Local audit and governance arrangements were followed at each site and the HQIP toolkit was used as an educational risk management tool in order to; promote adherence to local governance requirements and to reduce the potential of using unsafe data to confirm or change practice.

- Anonymised data was collected relating to the clinical management of 523 patients with PHPT.

\author{
Results: \\ Investigations; renal function (100\%), urinary calcium collection \\ (38\%), abdominal imaging (55\%), serum ACE (9\%). \\ Bone mineral density scanning (54\%), Average T-score Hip = -1.9 \\ Spine $=-1.7$ (only $27 \%$ of centres included forearm $=-2.1$ ). \\ Quality of life assessment $=0 \%$
}

Parathyroid imaging: requested as part of diagnostic work up in $82.5 \%$ of patients. Pre-operatively the imaging was not helpful in $33 \%$ of patients.

Indications for surgery;

- $73 \%$ of patients with a biochemical diagnosis of PHPT proceeded to have surgical parathyroidectomy

- Of these $81 \%$ fulfilled at least one of the criteria for surgery

- Of the remaining patients who did not have surgery $67 \%$ did fulfil at least one of the criteria

- $18 \%$ of the total patient population who would be indicated on the basis of the $3^{\text {rd }}$ international workshop guidelines were not operated and $14 \%$ who didn't qualify were operated.

Patients not having surgery;

Medical co-morbidities

Patient disinclination

Not referred for surgery because of negative imaging

Surgical disinclination because of negative imaging

'Watchful waiting'; annual serum calcium (96\%), renal function (90\%), vitamin D (79.5\%), DEXA scan (\%), use of Bisphosphonate $(31 \%)$, use of Cinacalcet $(8 \%)$, use of Oestogens ( \%).

\section{Key findings;}

- Inconsistencies in the management of PHPT which deviate from the recommendations of the $3^{\text {rd }}$ International workshop.

- No QoL, cognitive or psychological assessments

- Significant minority of patients not having surgery despite fulfilling the criteria or having surgery without fulfilling the requirements.

- Radiological tests as part of work up - implications on decision making for surgical referral or intervention

- Conservative Rx - some unnecessary / lack of testing

- Under-utilisation of bisphosphonates and Cinacalcet

\section{Conclusions}

There are major variations in UK practice. Suggestions include;

- We should cut down on the routine use of urinary calcium / creatinine clearance measurement

- No abdominal imaging unless a history of stone disease

- Failure to do enough DEXA scanning

- Endocrinologists should arguably not request parathyroid imaging

- Only dedicated endocrine surgeons with a high PTx workload should operate on patients with PHPT

- There is underuse of medical therapies

- Psychological and cognitive tests need to be validated and incorporated into clinical practice 